\title{
Molecular dynamics simulations of human E3 ubiquitin ligase Parkin
}

\author{
SHI QIU*, SHUN ZHU*, SHAN XU, YANYAN HAN, WEN LIU and JI ZUO \\ Department of Cellular and Genetic Medicine, School of Basic Medical Sciences, \\ Fudan University, Shanghai 200032, P.R. China
}

Received March 4, 2017; Accepted July 17, 2017

DOI: $10.3892 / \mathrm{mmr} .2017 .7140$

\begin{abstract}
Human E3 ubiquitin protein ligase parkin (Parkin) mediates mitophagy to maintain mitochondrial homeostasis. Parkin mutations are common genetic causes of early onset familial Parkinson's disease. The molecular mechanism of Parkin activation has been widely studied with emerging evidence suggesting an essential role of the phosphorylated (phospho)-ubiquitin interaction. However, the underlying mechanism of the phospho-ubiquitin interaction remains elusive. In the present study, replica exchange molecular dynamics simulations were performed to examine the conformational dynamics of Parkin in monomer and phospho-ubiquitin-bound states. In the Parkin monomer state, high structural flexibilities were observed in the majority of regions of Parkin particularly in the loop domain between the ubiquitin-like (UBL) and really interesting new gene (RING)0 domain. Binding of phospho-ubiquitin stabilizes the RING1/RING in between RING interface but destabilizes the RING1-UBL interface. Furthermore, using steered molecular dynamics simulations of Parkin mutations, it was demonstrated that salt bridge interactions contribute significantly to the interdomain interactions between the RING1 and UBL domain. Taken together, the results of the present study revealed the conformational dynamics of human full-length Parkin in monomer and phospho-ubiquitin-bound states, providing insights into designing potential therapeutics against Parkinson's disease.
\end{abstract}

\section{Introduction}

Parkinson's disease is a common neurodegenerative movement disorder with a prevalence of about 550 per 100,000

Correspondence to: Dr Wen Liu or Dr Ji Zuo, Department of Cellular and Genetic Medicine, School of Basic Medical Sciences, Fudan University, 130 DongAn Road, Shanghai 200032, P.R. China E-mail: liuwen@shmu.edu.cn

E-mail:jzuo@shmu.edu.cn

*Contributed equally

Key words: Parkin, Parkinson's disease, molecular dynamics, conformational dynamics, salt bridge at age 70 years (1). Recent genetic studies have identified mutations in E3 ubiquitin ligase Parkin that cause early onset familial Parkinson's disease (2). Parkin plays important roles in mitochondrial quality control by promoting the clearance of unhealthy mitochondria through mitophagy (3). In Parkin-mediated mitophagy, the PTEN induced putative kinase 1, PINK1 is first activated and translocated to the outer mitochondrial membrane. PINK1 activation in turn recruits and activates Parkin on OMM. Once activated, Parkin ubiquitinates a series of mitochondrial and cytosolic proteins, eventually leading to mitophagy $(4,5)$.

Although PINK1-induced Parkin activation has been widely studied (4-7), the precise mechanism of Parkin activation is not fully elucidated. Parkin consists of five structural domains: N-terminal ubiquitin-like (UBL) domain, RING0 domain, RING1 domain that binds E2 enzymes, IBR domain and RING2 domain that contains the active site $(8,9)$. It is generally recognized that before activation, these domains are arranged in such a way that Parkin is in an autoinhibited state: i) the UBL domain binds and occludes the E2 enzyme binding site in the RING1 domain; ii) the RING0 domain binds and occludes the catalytic site in the RING2 domain (9-11). Furthermore, recent biochemical and structural studies have revealed a role for phospho-ubiquitin (pUb) in Parkin activation: pUb binding may cause conformational changes in Parkin that release the UBL domain to activate Parkin (12-14). Notwithstanding, how pUb binding changes Parkin structure microscopically still remains elusive. Therefore, we conducted molecular dynamics (MD) simulations on two forms of Parkin in the current study, one of Parkin monomer and the other of Parkin-pUb complex.

Compared to experimental methods like X-ray crystallography, molecular dynamics simulations generate a structural ensemble of Parkininstead of just one structure (15).In this study, we used Replica-Exchange Molecular Dynamics (REMD) (16) simulations to examine the conformational dynamics of Parkin both in the monomer state and in pUb-bound state. For each state, 64 replicas were used in REMD simulations with $10 \mathrm{~ns}$ production time per replica, making the accumulated simulation time 1280 ns. Significant structural flexibilities were observed in Parkin monomer. pUb binding reduced the structural flexibilities in the RING1 and IBR domain. In addition, using steered molecular dynamics (SMD) simulations $(17,18)$ of Parkin monomer, we found that salt bridges 
play a crucial role in inter-domain interactions in Parkin. The binding of pUb causes conformational changes in Parkin that potentially disrupt these salt bridge interactions to facilitate the release of UBL domain during Parkin activation. This study provides structural insights into understanding the molecular mechanism of Parkin function and designing therapeutics for Parkinson's disease by modulating Parkin activities.

\section{Materials and methods}

System building. Parkin is a 465 amino-acid protein that consists of four domains: N-terminal ubiquitin-like (UBL) domain (residues 1-76); RING0 domain (residues 145-215); RING1 domain (residues 237-292) that binds to E2 ubiquitin-conjugating enzyme; IBR domain (residues 327-378); RING2 domain that mediates the enzyme's catalytic activity (415-465). There exist several crystal structures of Parkin in Protein Data Bank (http://www.rcsb.org). The loop domain between the UBL and RING0 domain (residue 77-144) is unresolved in all crystal structures of Parkin. To build the full-length Parkin model, Modeller 9.15 (19) was used and the PDB codes for the four templates listed below were used: $4 \mathrm{I} 1 \mathrm{~F}(10), 5 \mathrm{C} 9 \mathrm{~V}$ (14), 5C1Z (20), 4WZP (21). For the model of Parkin-pUb complex, a crystal structure of Parkin-pUb complex from Pediculus humanus (PDB code: 5CAW) were used as a template (14).

Simulation setup. All REMD simulations were performed in Gromacs 5.0.5 (22,23). The force field GROMOS54a7 (24) was used. The parameters for phosphorylated serine in $\mathrm{pUb}$ were obtained from Vienna-PTM 2.0 (25). The rectangular simulation box dimensions were set up to be the same for the Parkin monomer system and the Parkin-pUb system: A $20 \AA$ buffer was used to enclose both systems. Water molecules were simulated using the SPC model. The time step of the simulation was set to be 2.5 fs. First, steepest descent energy minimization was performed for 1,000 steps, followed by a gradual heat up of the system in NPT ensemble from $50 \mathrm{~K}$ to $250 \mathrm{~K}$. Then equilibration was performed at each replica exchange temperature for $100 \mathrm{ps}$. The equilibrated structures were used in subsequent REMD simulations. The REMD simulation contains 64 replicas from $298 \mathrm{~K}$ to $373 \mathrm{~K}$, with temperatures generated from REMD temperature prediction server (26). NPT simulation of each replica was performed for $10 \mathrm{~ns}$ with exchanges attempted every $2.5 \mathrm{ps}$. An extension of the Parkin-pUb system to $18 \mathrm{~ns}$ per replica was also performed. Replicas at all 64 temperatures for Parkin or Parkin-pUb simulations were concatenated and used for REMD analysis in this study unless specified otherwise.

RMSF calculations. Root mean square fluctuations or RMSFs were calculated using also the initial structure of Parkin as reference. By fitting Parkin structures in all replicas to the reference structure, a residue-based RMSF can be obtained.

Secondary structure calculations. To obtain the secondary structure composition of the loop domain, DSSP programs (27) were used on Parkin structures extracted from all replicas in Parkin monomer or Parkin-pUb systems.
Principal component analysis. Principal component analysis (PCA) uses an orthogonal transformation to extract major features called principal components from a dynamic complex system. g_covar utility was used to generate the covariance matrix of trajectory coordinates of Parkin monomer with eigenvectors and eigenvalues calculated. Then the simulation trajectories of both Parkin monomer and Parkin-pUb were projected onto the first two eigenvectors derived from Parkin monomer simulations using g_anaeig utility. The resulting PC1 and PC2 were used for subsequent plotting.

Distance measurements. Inter-domain distances were defined as the distance between centers of geometry of two domains. Two inter-domain distances were simultaneously measured and plotted for RING1-UBL and RING1-IBR: $\mathrm{d}_{\text {RING1-UBL }}$ and $\mathrm{d}_{\text {RING1-IBR }}$. The resulting dot distributions are then converted to probability distribution 2D surface with the negative natural logarithm of probabilities being plotted on the $\mathrm{Z}$ direction.

Steered molecular dynamics simulations. Steered molecular dynamics (SMD) simulations were prepared using VMD 1.9.3 (28) and performed in NAMD 2.12 (29). Specifically, an external force was applied onto the UBL domain to pull the UBL domain away from the Parkin core at a constant velocity until it is released from the Parkin core. At every time step during the SMD simulation, the external forces applied were recorded, the magnitude of which is proportional to the strength of the interaction that are being disrupted. $\mathrm{C} \alpha$ atoms of residues 1 to 76 (the UBL domain) were configured to be SMD atoms, i.e., atoms being pulled. $\mathrm{C} \alpha$ atoms of residues 145 to 465 (Parkin core) were configured to be fixed atoms, which are being fixed during pulling. A constant velocity of $0.0005 \AA /$ step was used for the pulling. The virtual spring that connects the dummy atom and the UBL domain has a spring constant of $7 \mathrm{kcal} / \mathrm{mol} / \AA 2$. The pulling direction was defined to be the normalized direction from the center of mass of fixed atoms to the center of mass of SMD atoms. SMD simulations were performed in wild-type and mutant Parkin for $1 \mathrm{~ns}$ for each system. In addition, SMD simulations were also performed with a pulling velocity of $0.00025 \AA$ Astep for 2 ns to examine the reproducibility of the SMD results.

\section{Results}

Construction of full-length Parkin structure. The crystal structures of Parkin in protein data bank all have a large fraction of missing residues at the loop region between the UBL domain and RING0 domain. To build the structure of full-length human Parkin and Parkin-pUb (Fig. 1), we used five templates with PDB codes 5C1Z, 5C9V, 4I1F, 4WZP, and $5 \mathrm{CAW}$. The loop region was modeled and refined to make sure that the location of the loop looks reasonable. Note that the Parkin-pUb complex model was firstly built and the Parkin monomer model was constructed by simply removing $\mathrm{pUb}$ from the complex. This procedure was taken to ensure the quality of the comparison between Parkin monomer simulations and Parkin-pUb simulations.

Structural fluctuations in Parkin. To examine the conformational dynamics of Parkin, structural snapshots in replica 


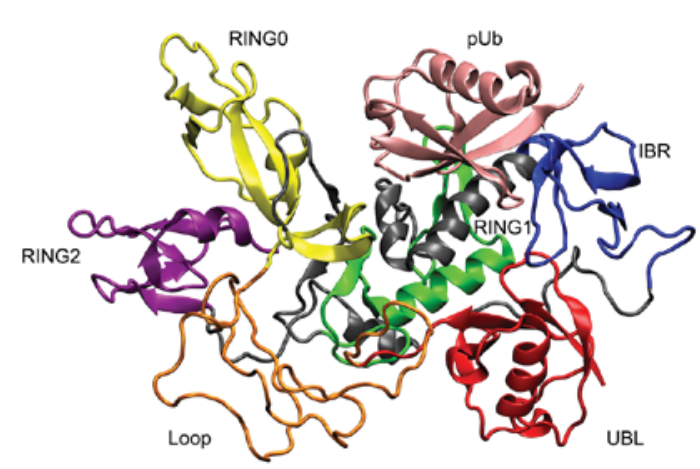

Figure 1. Construction of full-length Parkin structure. Note that Parkin monomer and Parkin-pUb complex have the same initial structural coordinates for Parkin. Red represents the UBL domain (residues 1-76); orange represents the loop domain (residues 77-144); yellow represents the RING0 domain (residues 145-215); green represents the RING1 domain (residues 237-292); blue represents the IBR domain (residues 327-378); purple represents the RING2 domain (residues 415-465) and pink represents pUb. Inter-domain linkers are colored in dark gray. UBL, ubiquitin-like; RING0, really interesting new gene; $\mathrm{pUb}$, phospho-ubiquitin.

exchange molecular dynamics simulations were combined for subsequent analysis. RMSF analysis was performed for all atoms of full-length Parkin in Parkin monomer system and Parkin-pUb system, respectively. Structure fluctuations of Parkin follow a similar pattern in Parkin monomer and Parkin-pUb (Fig. 2A), indicating that intrinsic dynamics dominates the conformational landscape of Parkin. The RMSF results agree well with that of a previous simulation study of Parkin (30).

Overall, considerable fluctuations were observed in full-length Parkin. For example, about $93 \%$ of Parkin residues exhibits RMSFs higher than $3 \AA$ in the Parkin monomer. With that said, different regions of Parkin exhibit varied flexibilities: The peripheral and solvent-exposed region (ribbons colored in white and red in Fig. 2B, C) exemplified by the loop domain appears to be noticeably more flexible than the core and buried region (ribbons colored in blue in Fig. 2B, C). Interestingly, the loop domain seems to harbor regions with distinct flexibilities: The regions around residue 87 and residue 134 are highly flexible while the region around residue 118 is comparatively rigid. A further analysis of the secondary structure composition of the loop domain throughout the simulation revealed that the region around residue 118 preferred to adopt $\beta$-sheet while the regions around residue 87 and 134 prefer random coil (Fig. 3A), which is consistent with their relative flexibility. Comparing Parkin monomer and Parkin-pUb complex, the latter seems to favor a higher fraction of $\beta$-sheet structures around residue 80 and 110 as well as a higher fraction of $\alpha$-helix structures around residue130 (Fig. 3A and B). In addition, the variations of secondary structure in three replicas at representative temperatures, $298 \mathrm{~K}, 335.66 \mathrm{~K}$, and $373.53 \mathrm{~K}$ were also measured, indicating high flexibility of the loop domain especially at high temperatures (Fig. 3C).

Comparing RMSFs in Parkin monomer and Parkin-pUb, we observed differential effects of $\mathrm{pUb}$ on Parkin domains: for the UBL, RING0, and RING2 domains, pUb binding made little difference in terms of structural fluctuations; for the RING1 and IBR domains, however, pUb binding significantly dampened the structural fluctuations (Fig. 2A). The dampening effects of pUb can also be observed in the RMSD

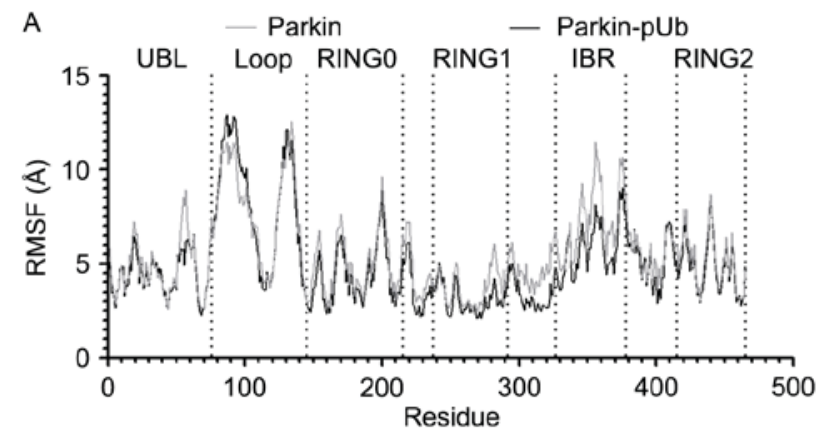

B

C

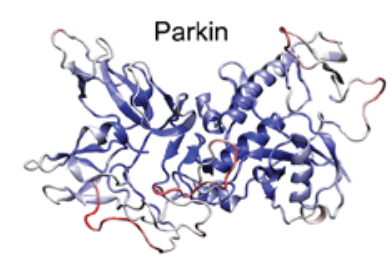

Parkin-pUb

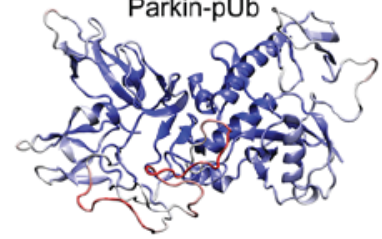

Figure 2. Structural fluctuations in Parkin. (A) RMSF of Parkin in Parkin monomer and Parkin-pUb simulations. The RMSF curves are divided into sections based on the following domains: the UBL domain (residues 1-76); the loop domain (residues 77-144); the RING0 domain (residues 145-215); the RING1 domain (residues 237-292); the IBR domain (residues 327-378); the RING2 domain (residues 415-465). Gray and black solid lines represent Parkin monomer and Parkin-pUb simulations, respectively. RMSF mappings to full-length Parkin are shown for Parkin monomerm (B) and Parkin-pUb (C) system. BWR (Blue-White-Red) gradient is used, in which blue, white and red represent low, medium and high RMSFs, respectively. RMSF, root mean square fluctuation; UBL, ubiquitin-like; RING0, really interesting new gene; pUb, phospho-ubiquitin.

distributions of Parkin structures at different representative temperatures (Fig. 4A). The discrepancy can be potentially explained by the fact that pUb makes direct physical contact with the RING1 and IBR domains in Parkin in Parkin-pUb binding. The effects of pUb observed here are consistent with the dual role of pUb discovered in an earlier study (30): Competitive binding with the UBL domain and stabilization of the RING1 and IBR domains.

To understand the convergence of the simulations, we examined the self- consistency of Parkin structures by measuring RMSF using the first half and the second half of the trajectory, respectively. The first half and second half of the trajectory exhibited considerable similarity (Fig. 4B), indicating that Parkin monomer and Parkin-pUb simulation have obtained decent self-consistency.

Correlated domain motions in Parkin. To identify domain movements in Parkin that might be functionally relevant, correlated motions or principal components (PCs) were extracted from structural ensembles of Parkin in all replicas using principal component analysis (PCA). The top-ranked two most dominant principal components, PC1 and PC2, were obtained for Parkin in Parkin monomer and Parkin-pUb simulations, respectively. Generally speaking, PC1-PC2 exhibits a more compact landscape in Parkin-pUb simulations (Fig. 5A, red surfaces) compared to that in Parkin monomer simulations (Fig. 5A, blue surfaces). Although the PC1-PC2 landscapes in Parkin-pUb simulations and Parkin monomer simulations overlap, they each occupy unique regions on the two-dimensional surface. 

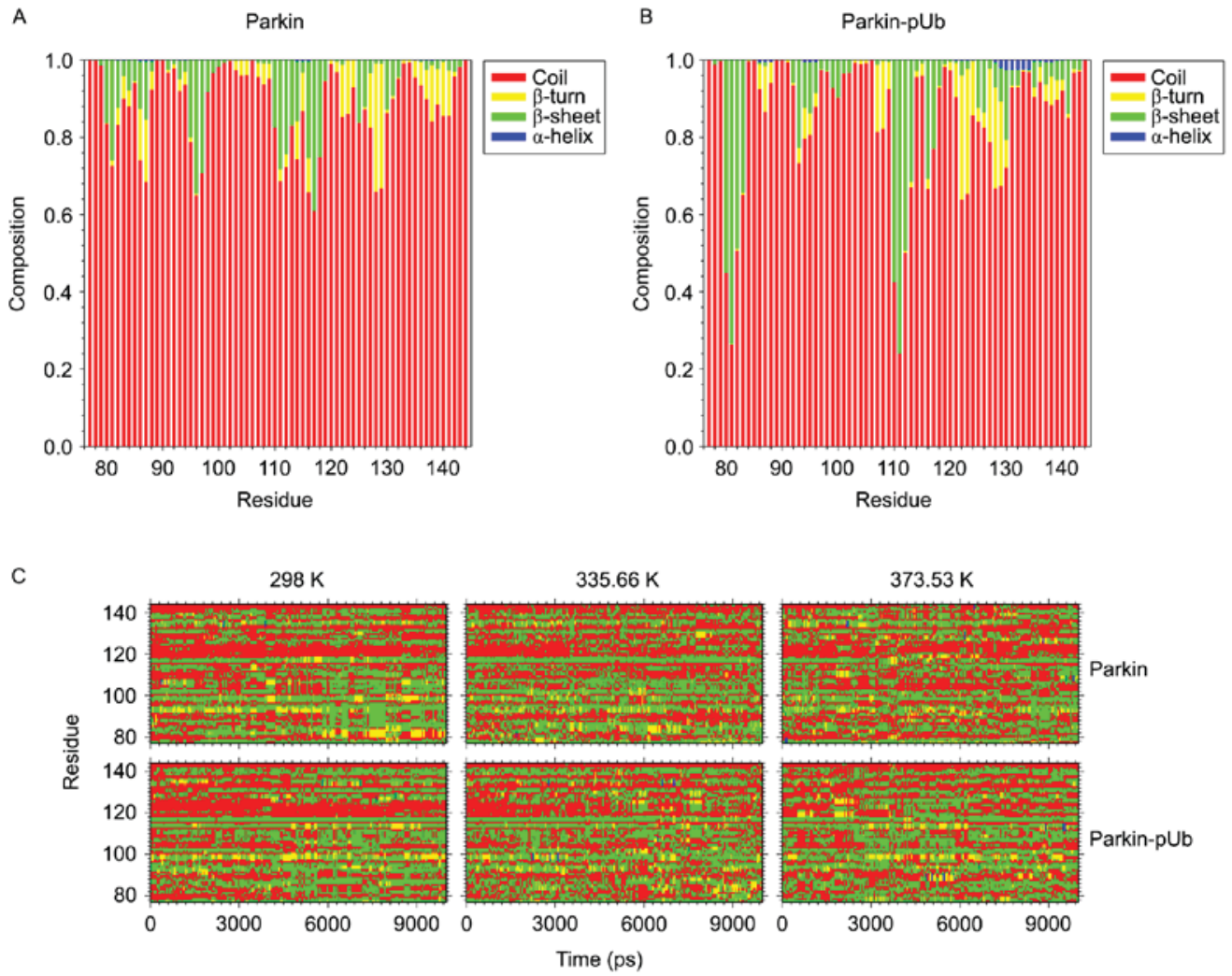

Figure 3. Compositions of secondary structures for the loop domain in Parkin monomer (A) and Parkin-pUb (B) simulations. To obtain the secondary structure compositions, structures of Parkin at all replicas were collected and subject to secondary structure alignments. Red represents random coil, yellow represents $\beta$-turn, green represents $\beta$-sheet and blue represents $\alpha$-helix. (C) The variations of secondary structure in the simulation were shown for three replicas at representative temperatures, $298 \mathrm{~K}, 335.66 \mathrm{~K}$, and $373.53 \mathrm{~K}$. Red represents random coil, yellow represents $\beta$-turn, green represents $\beta$-sheet and blue represents $\alpha$-helix.
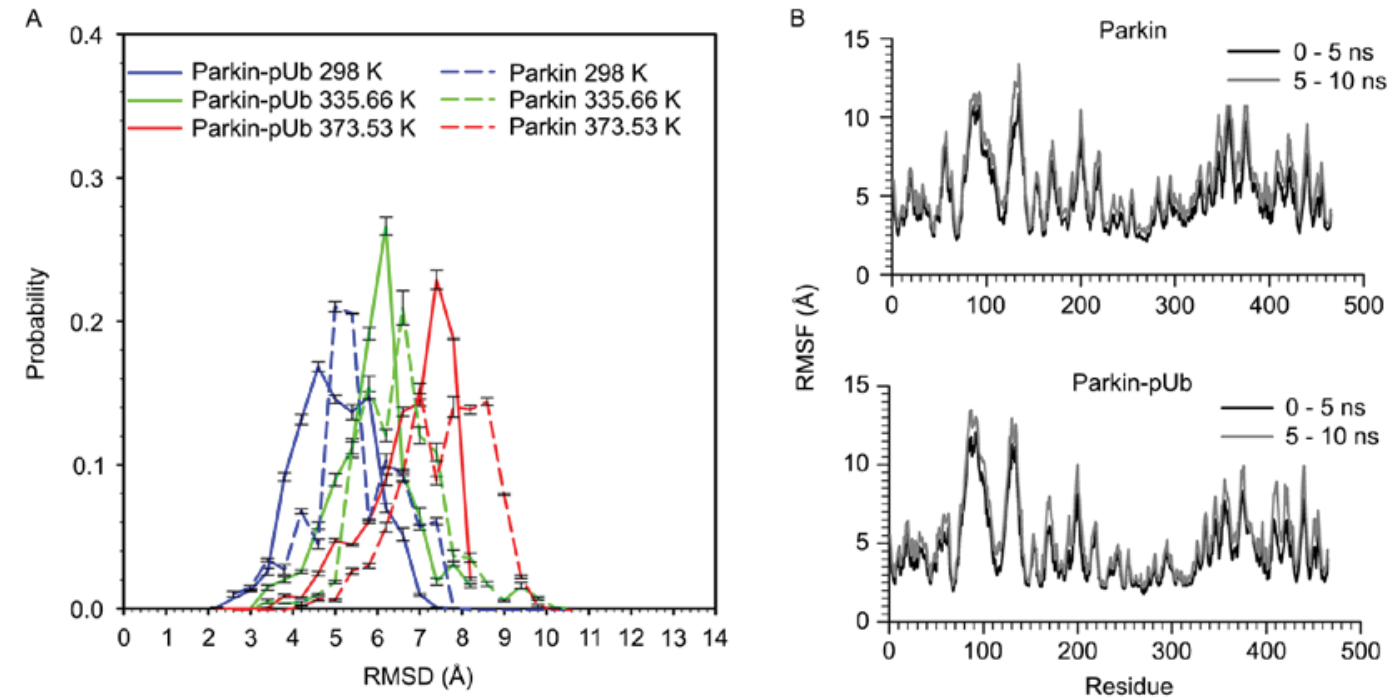

Figure 4. Structural variations of Parkin in replicas at representative temperatures and in sub-sampled trajectories. (A) RMSD distributions for replicas at three representative temperatures. Dashed and solid lines represent Parkin monomer and Parkin-pUb simulations, respectively. RMSDs were calculated by fitting backbones of the full-length Parkin to the initial structure of Parkin. The error bars for RMSD calculations was obtained from bootstrapping method: Half of data points were randomly resampled for 3 times independently to obtain the standard error. (B) RMSFs calculated using the first and the second half of the trajectory. The first (0-5 ns, shown in black) and second half (5-10 ns, shown in gray) of the trajectory for all 64 replicas were used in both Parkin monomer and Parkin-pUb simulations.

Principal component analysis captured the essential dynamics of the whole Parkin protein but not contributions from individual domains. To examine the effects of pUb on the dynamics of Parkin domains, the RING1, UBL, and IBR domains were selected for distance analysis due to their proximities to $\mathrm{pUb}$. Specifically, inter-domain distances were 
A

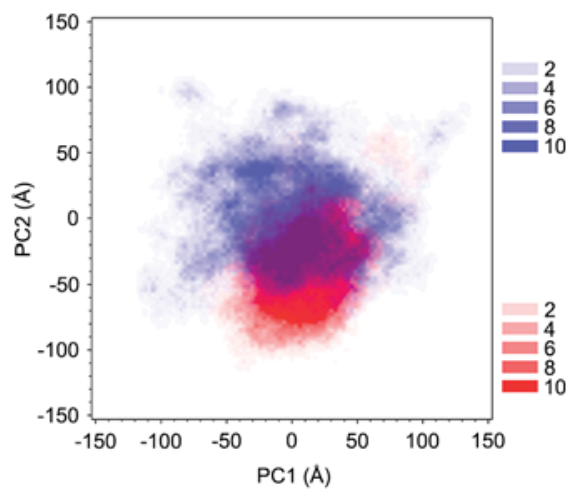

B

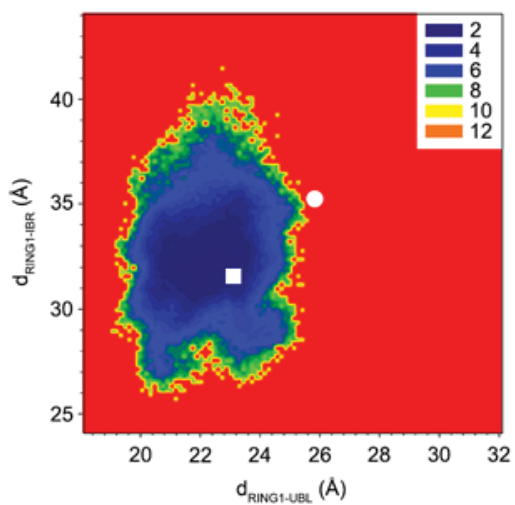

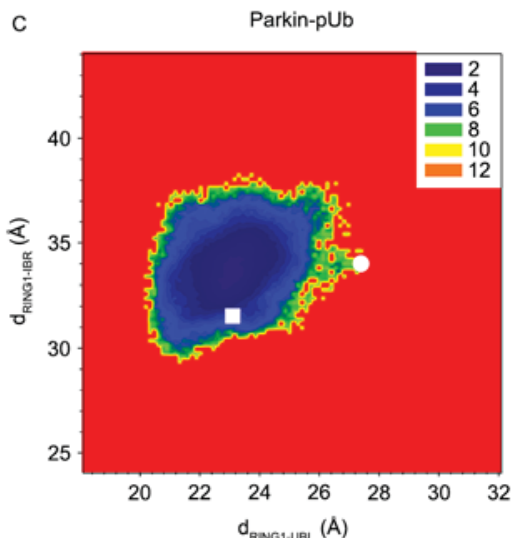

Figure 5. Domain movements in Parkin. (A) Density plot of principal components, PC1 vs. PC2. PC1 and PC2 were extracted from trajectories of Parkin Ca atoms in Parkin monomer and Parkin-pUb simulations. Blue and red surfaces represent Parkin monomer system and Parkin-pUb system, respectively. Both surfaces are colored with transparent color. (B) 2D distance probability surface for the Parkin monomer system. (C) 2D distance probability surface for the Parkin-pUb system. The color in (B) and (C) represents the negative natural logarithm of probabilities. All inter-domain distances were measured between centers of geometry of domains. The white square represents the initial structure and the white circle represents the structure with maximum distance between the RING1 and UBL domain.

measured between the RING1 and UBL domains as well as between the RING1 and IBR domains for all snapshots in the simulations to construct a two-dimensional distance probability surface. A noticeably expanded conformational space was explored in the MD simulations starting from the initial structure (Fig. 5B, C). In addition, pUb differentially influenced the two inter-domain distances: the excursion along RING1-IBR axis seems to be reduced (the distance range changing from 25.7-41.5 $\AA$ in Parkin monomer to 29.4-38.3 in Parkin-pUb); the excursion along RING1-UBL axis seems to be slightly increased (range changing from 19.0-25.8 $\AA$ in Parkin monomer to 20.2-27.4 $\AA$ in Parkin-pUb). These results suggest that pUb stabilizes the RING1-IBR interface and destabilizes the RING1-UBL interface.

Changes of salt bridge distances in inter-domain motions. We have shown that pUb binding can affect the conformational dynamics and inter-domain motions in Parkin. However, it is not well appreciated how pUb binding eventually leads to the release of the UBL domain during Parkin activation. One possibility is by decreasing the 'affinity' between the UBL domain and the RING1 domain. Previous studies have suggested the role of salt bridges in maintaining inter-domain interactions in Parkin between the UBL domain and the RING1 domain (20). Therefore, we examined the status of three salt bridges at the RING1-UBL interface when RING1-UBL distance is at its maximum (Fig. 5B, 5C, circles): ARG6-ASP274, ARG42-ASP262, and ARG72-ASP262.

In the initial structure, all three salt bridges are stably maintained (Fig. 6A). In Parkin monomer simulations, salt bridges ARG42-ASP262 and ARG72-ASP262 are lost (salt bridge distance changing from $4.33 \AA$ to $12.39 \AA$ for ARG42-ASP262, from $3.95 \AA$ to $12.14 \AA$ for ARG72-ASP262) (Fig. 6B). In Parkin-pUb simulations, salt bridges ARG42-ASP262 and ARG72-ASP262 are also lost (salt bridge distance changing from $4.33 \AA$ to $17.46 \AA$ for ARG42-ASP262, from $3.95 \AA$ to $19.33 \AA$ for ARG72-ASP262) (Fig. 6C). In contrast, salt bridge ARG6-ASP274 is stably maintained in both Parkin monomer and Parkin-pUb simulations (salt bridge distance changing

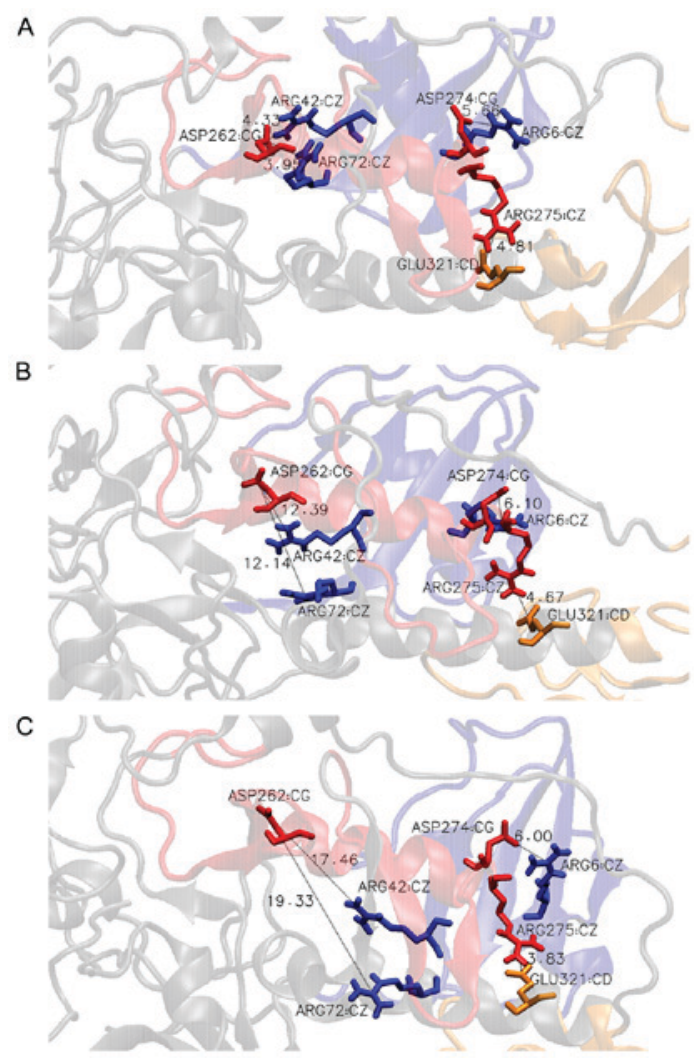

Figure 6. Structures of inter-domain salt bridges. The transparent red, blue, and orange ribbons represent the RING1 domain, UBL domain and IBR domain, respectively. The same color scheme also applies to salt bridge residues shown in licorice representation. (A) Salt bridges in the initial structure of Parkin. (B) Salt bridges in the structure with maximum distance between the RING1 and UBL domain in Parkin monomer simulations. (C) Salt bridges in the structure with maximum distance between the RING1 and UBL domain in Parkin-pUb simulations. The salt bridge distance was defined as the carbon-carbon distance between ARG:CZ in UBL domain and ASP:CG/GLU:CD in the RING1/IBR domain. UBL, ubiquitin-like; RING0, really interesting new gene domain; $\mathrm{pUb}$, phospho-ubiquitin.

only slightly from $5.66 \AA$ to around $6 \AA$ ) (Fig. 6B, C), suggesting that salt bridge ARG6-ASP274 is dominant in 

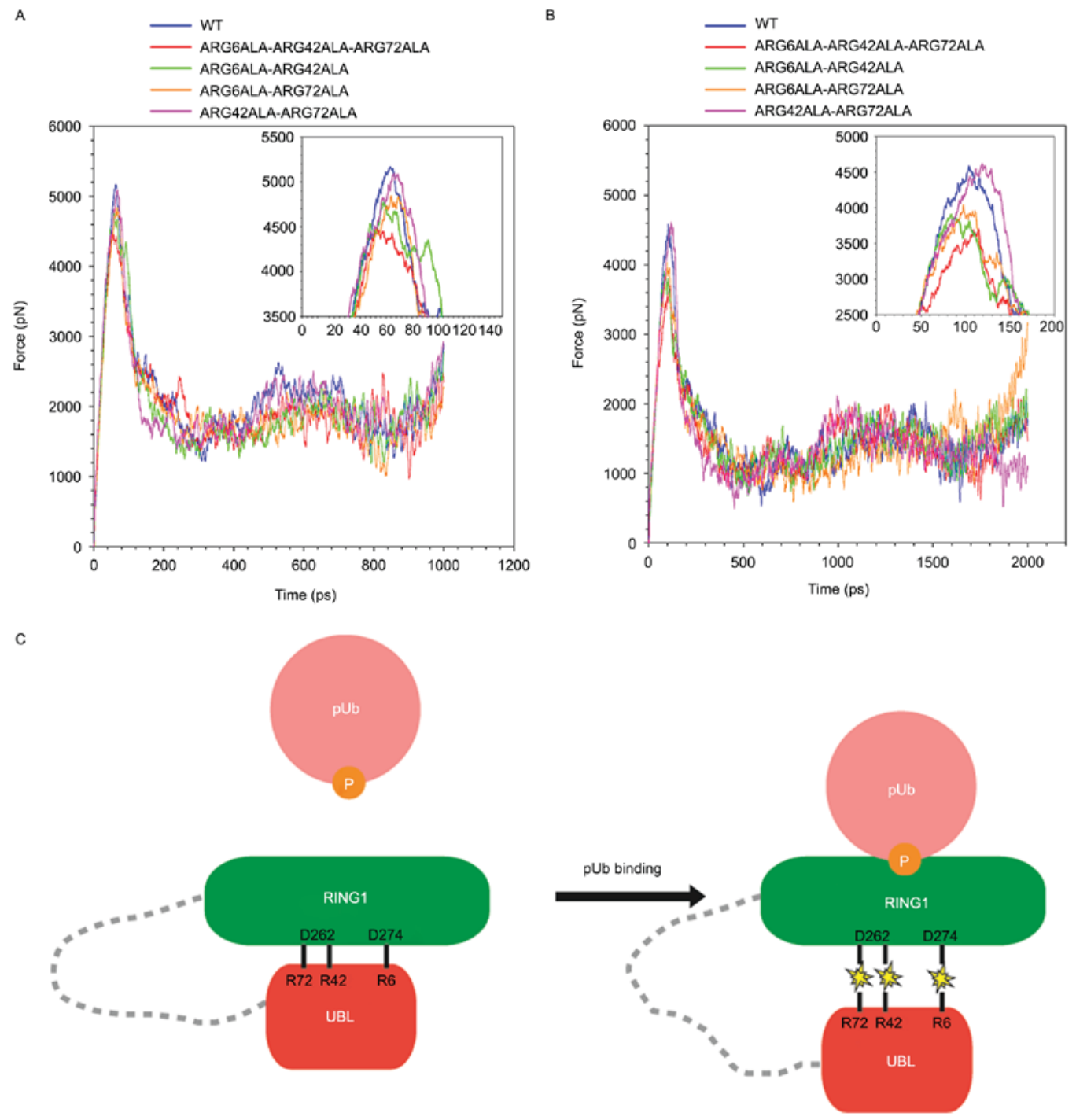

Figure 7. Salt bridges are important for inter-domain interactions between the RING1 and UBL domain. Force vs. time plots for SMD simulations in wild-type and mutant Parkin performed in two conditions: (A) pulling velocity of $0.0005 \AA /$ step for 1ns; (B) pulling velocity of $0.00025 \AA /$ step for 2 ns. One triple mutant and three double mutants were set up for SMD simulations. The inner panel is an enlarged version of the force barrier. (C) A model for pUb-induced Parkin activation, highlighting the breaking of salt bridges upon pUb binding: ARG6-ASP274, ARG42-ASP262, and ARG72-ASP262. RING0, really interesting new gene; pUb, phospho-ubiquitin; UBL, ubiquitin-like.

maintaining RING1-UBL interactions. As a control, we also measured the distance for a salt bridge at the RING1-IBR interface, ARG275-GLU321. While this salt bridge seems quite stable in both Parkin monomer and Parkin-pUb simulations, it seems to be more stable in Parkin-pUb simulations (salt bridge distance is $4.67 \AA$ and $3.83 \AA$ in Parkin monomer and Parkin-pUb simulations) (Fig. 6B, C), in agreement with the distance probability surface results shown above.

Crucial roles of salt bridges in inter-domain interactions. To further investigate the contributions of salt bridges ARG6-ASP274, ARG42-ASP262, and ARG72-ASP262 in maintaining inter-domain interactions between the RING1 and UBL domains, we mutated critical salt bridge residues to alanine at the RING1-UBL interface in Parkin monomer and measured the changes of RING1-UBL 'affinity' using SMD simulations. In SMD simulations, we pulled the UBL domain away from the RING1 domain and measured the resistance force, which is proportional to the RING1-UBL 'affinity'. In our $1 \mathrm{~ns}$ SMD simulations, a force barrier of $5170 \mathrm{pN}$ at around 70 ps was observed (Fig. 7A, blue line) in wild-type Parkin, which corresponds to the time when the UBL domain started to dissociate from the Parkin core, i.e., the RING1 domain. When all three arginine residues were mutated to alanine, the force barrier underwent a $13 \%$ decrease, from $5170 \mathrm{pN}$ to $4494 \mathrm{pN}$ (Fig. 7A, red lines), indicating a significant contribution from salt bridges for RING1-UBL interactions. R42A-R72A double mutants, however, showed only slightly decreased force barrier: the force barrier underwent a $1.3 \%$ decrease from $5170 \mathrm{pN}$ to $5100 \mathrm{pN}$ (Fig. 7A, purple lines). In addition, ARG6-defective double mutants showed noticeably lowered force barriers: R6A-R42A and R6A-R72A went down to $4775 \mathrm{pN}$ and $4847 \mathrm{pN}$, respectively (Fig. 7A, green and orange lines). To ensure the reproducibility of SMD results, we also performed 2 ns SMD simulations with half of the pulling velocity as in $1 \mathrm{~ns}$ SMD simulation and observed the same trend in terms of the height of the force barriers as in $1 \mathrm{~ns}$ SMD simulations (Fig. 7B). The above results indicate 
that the salt bridge mediated by ARG6 has more contributions to RING1-UBL interactions among the three salt bridges.

\section{Discussion}

The number of simulation studies of full-length Parkin is quite limited partly due to the difficulties in modeling the unresolved loop domain $(30,31)$. To our knowledge, our study reported here is the first MD simulation study of full-length Parkin. Considering the unresolved loop domain is lengthy (nearly 70 amino acids), to build its structural model is quite challenging because multiple conformations of the loop may exist. One needs to bear in mind that the model of the loop domain constructed in our study is just one instance of many potentially favorable conformations. Interestingly, we found that although most regions in the loop domain stayed disordered during the simulations, some regions preferentially formed secondary structures such as $\beta$-sheet and $\alpha$-helix.

pUb binding is considered to cause conformational changes in Parkin that changes Parkin from the autoinhibited state into the open and active state (32). Although a full transition event was not observed in MD simulations due to difficulties in sampling, indicators of the transition events were found. First, in Parkin-pUb system, the RING1-UBL inter-domain distance range was expanded compared to Parkin monomer. Second and more importantly, a destabilization effect of pUb on the RING1-UBL interface was observed, which was found to be potentially related to the stability of several salt bridges at the RING1-UBL interface. Although salt bridge interactions at the RING1-UBL interface are important for maintaining RING1-UBL interactions, other types of interactions such as hydrogen bond and hydrophobic interactions may also have roles in the process, which awaits further investigations.

In the present study, we performed MD simulation studies of Parkin in monomer and pUb-bound states. We propose a potential model for pUb-induced Parkin activation (Fig. 7C). First, pUb binds the autoinhibited Parkin and forms strong electrostatic interactions with the RING1 domain, causing conformational changes of the RING1 domain. Second, conformational changes of the RING1 domain destabilize the RING1-UBL interface by disrupting crucial salt bridge interactions at the interface: ARG6-ASP274, ARG42-ASP262, and ARG72-ASP262. Lastly, the UBL domain is released from the RING1 domain as well as the Parkin core, eventually leading to Parkin activation. Certain Parkin mutations may prevent Parkin from being activated, which is a potential cause of Parkinson's disease. By perturbing the salt bridges at the UBL-RING1 interface using rationally designed peptides and small molecules, we may facilitate the release of UBL domain to reactivate Parkin. Therefore, our study provides insights into designing potential therapeutics for Parkinson's disease.

\section{Acknowledgements}

The present study was supported by grants obtained from the National Natural Science Foundation of China (grant no. 81501096), the Key Basic Research Project of Science and Technology Commission of Shanghai Municipality (grant no. 13JC1401100/101) and the Open Project of State Key Laboratory of Medical Neurobiology (grant no. SKLMN2014003).

\section{References}

1. Louis ED, Mayer SA and Rowland LP: Merritt's Neurology. 13th edition. Lippincott and Williams \& Wilkins, Philadelphia, 2015.

2. Tan EK and Skipper LM: Pathogenic mutations in Parkinson disease. Hum Mutat 28: 641-653, 2007.

3. Youle RJ and Narendra DP: Mechanisms of mitophagy. Nat Rev Mol Cell Biol 12: 9-14, 2011.

4. Jin SM and Youle RJ: PINK1-and Parkin-mediated mitophagy at a glance. J Cell Sci 125: 795-799, 2012.

5. Scarffe LA, Stevens DA, Dawson VL and Dawson TM: Parkin and PINK1: Much more than mitophagy. Trends Neurosci 37: 315-324, 2014.

6. Sun Y, Vashisht AA, Tchieu J, Wohlschlegel JA and Dreier L: Voltage-dependent Anion Channels (VDACs) recruit parkin to defective mitochondria to promote mitochondrial autophagy. J Biol Chem 287: 40652-40660, 2012.

7. Burchell VS, Nelson DE, Sanchez-Martinez A, Delgado-Camprubi M, Ivatt RM, Pogson JH, Randle SJ, Wray S, Lewis PA, Houlden H, et al: The Parkinson's disease-linked proteins Fbxo7 and Parkin interact to mediate mitophagy. Nat Neurosci 16: 1257-1265, 2013.

8. Trempe JF and Fon EA: Structure and function of Parkin, PINK1 and DJ-1, the three musketeers of neuroprotection. Front Neurol 4: 38, 2013.

9. Trempe JF, Sauvé V, Grenier K, Seirafi M, Tang MY, Ménade M, Al-Abdul-Wahid S, Krett J, Wong K, Kozlov G, et al: Structure of parkin reveals mechanisms for ubiquitin ligase activation. Science 340: 1451-1455, 2013.

10. Riley BE, Lougheed JC, Callaway K, Velasquez M, Brecht E, Nguyen L, Shaler T, Walker D, Yang Y, Regnstrom K, et al: Structure and function of Parkin E3 ubiquitin ligase reveals aspects of RING and HECT ligases. Nat Commun 4: 1982, 2013.

11. Ham SJ, Lee SY, Song S, Chung JR, Choi S and Chung J: Interaction between RING1 (R1) and the ubiquitin-like (UBL) domains is critical for the regulation of Parkin activity. J Biol Chem 291: 1803-1816, 2016.

12. Chaugule VK, Burchell L, Barber KR, Sidhu A, Leslie SJ, Shaw GS and Walden H: Autoregulation of Parkin activity through its ubiquitin-like domain. Embo J 30: 2853-2867, 2011.

13. Wauer T and Komander D: Structure of the human Parkin ligase domain in an autoinhibited state. Embo J 32: 2099-2112, 2013.

14. Wauer T, Simicek M, Schubert A and Komander D: Mechanism of phospho-ubiquitin-induced PARKIN activation. Nature 524: 370-374, 2015.

15. Karplus M and McCammon JA: Molecular dynamics simulations of biomolecules. Nat Struct Biol 9: 646-652, 2002

16. Sugita Y and Okamoto Y: Replica-exchange molecular dynamics method for protein folding, Chem Phys Lett 314: 141-151, 1999.

17. Lu H, Isralewitz B, Krammer A, Vogel V and Schulten K: Unfolding of titin immunoglobulin domains by steered molecular dynamics simulation. Biophys J 75: 662-671, 1998.

18. Isralewitz B, Gao M and Schulten K: Steered molecular dynamics and mechanical functions of proteins. Curr Opin Struct Biol 11: 224-230, 2001

19. Webb B and Sali A: Comparative Protein Structure Modeling Using MODELLER. Curr Protoc Bioinformatics 86: 5.6.1-5.6.37, 2016.

20. Kumar A, Aguirre JD, Condos TE, Martinez-Torres RJ, Chaugule VK, Toth R, Sundaramoorthy R, Mercier P, Knebel A, Spratt DE, et al: Disruption of the autoinhibited state primes the E3 ligase parkin for activation and catalysis. EMBO J 34: 2506-2521, 2015

21. Wauer T, Swatek KN, Wagstaff JL, Gladkova C, Pruneda JN, Michel MA, Gersch M, Johnson CM, Freund SM and Komander D: Ubiquitin Ser65 phosphorylation affects ubiquitin structure, chain assembly and hydrolysis. EMBO J 34: 307-325, 2015.

22. Hess B, Kutzner C, van der Spoel D and Lindahl E: GROMACS 4: Algorithms for highly efficient, load-balanced and scalable molecular simulation. J Chem Theory Comput 4: 435-447, 2008.

23. Pronk S, Páll S, Schulz R, Larsson P, Bjelkmar P, Apostolov R, Shirts MR, Smith JC, Kasson PM, van der Spoel D, et al: GROMACS 4.5: A high-throughput and highly parallel open source molecular simulation toolkit. Bioinformatics 29: 845-854, 2013. 
24. Schmid N, Eichenberger AP, Choutko A, Riniker S, Winger M, Mark AE and van Gunsteren WF: Definition and testing of the GROMOS force-field versions 54A7 and 54B7. Eur Biophys J 40: 843-856, 2011.

25. Petrov D, Margreitter C, Grandits M, Oostenbrink C and Zagrovic B: A systematic framework for molecular dynamics simulations of protein post-translational modifications. PLoS Comput Biol 9: e1003154, 2013.

26. Patriksson A and van der Spoel D: A temperature predictor for parallel tempering simulations. Phys Chem Chem Phys 10: 2073-2077, 2008.

27. Kabsch W and Sander C: Dictionary of protein secondary structure: Pattern recognition of hydrogen-bonded and geometrical features 22: 2577-2637, 1983

28. Humphrey W, Dalke A and Schulten K: VMD: Visual molecular dynamics. J Mol Graph 14: 33-38, 1996.
29. Phillips JC, Braun R, Wang W, Gumbart J, Tajkhorshid E, Villa E, Chipot C, Skeel RD, Kalé L and Schulten K: Scalable molecular dynamics with NAMD. J Comput Chem 26: 1781-1802, 2005.

30. Walinda E, Morimoto D, Sugase K and Shirakawa M: Dual function of phosphoubiquitin in E3 activation of parkin. J Biol Chem 291: 16879-16891, 2016.

31. Tomoo K, Mukai Y, In Y, Miyagawa H, Kitamura K, Yamano A, Shindo $\mathrm{H}$ and Ishida T: Crystal structure and molecular dynamics simulation of ubiquitin-like domain of murine parkin. Biochim Biophys Acta 1784: 1059-1067, 2008.

32. Sauvé V, Lilov A, Seirafi M, Vranas M, Rasool S, Kozlov G, Sprules T, Wang J, Trempe JF and Gehring K: A Ubl/ubiquitin switch in the activation of Parkin. EMBO J 34: 2492-2505, 2015. 